\title{
Hydrodynamic permeability prediction for flow through 2D arrays of rectangles
}

\author{
M. Cloete \& J. P. du Plessis \\ Department of Applied Mathematics, University of Stellenbosch, \\ South Africa
}

\begin{abstract}
In the present paper a model to predict the hydrodynamic permeability of viscous flow through an array of squares is generalized to include flow through arrays of rectangles of any aspect ratio. This involves different channel widths in the streamwise and the transverse flow directions. It is shown how, with the necessary care taken during description of the interstitial geometry, a volume averaged approach can be used to obtain results identical to a direct method. Insight into the physical situation is gained during the modelling of the two-dimensional interstitial flow processes and resulting pressure distributions and this may prove valuable when the volume averaging method is applied to more complex three-dimensional cases. The analytical results show close correspondence to numerical calculations except in the higher porosity range for which a more realistic model is needed. Keywords: porous media, volume averaging, hydrodynamic permeability, rectangles.

\section{Introduction}

Apart from the spatial dimension of the microstructure, the analytical result involves two parameters, the first of which relates to the extent of staggeredness that a fluid particle experiences on its way downstream. The second parameter introduced is a measure of aspect ratio of the rectangles which will allow us to vary the length of the transverse channels. In this paper the influence of these two parameters on the hydrodynamic permeability will be discussed.
\end{abstract}

\section{Direct analytical modelling}

Following Firdaouss and Du Plessis [1], the solid phase and the unit cell are represented by rectangles of the same aspect ratio. This was done to incorporate 
situations where the interstitial velocity in the channels parallel to the streamwise direction differs from that in the transverse channels, while maintaining notational simplicity. The fluid-solid interface parallel to the streamwise direction is denoted by $d s_{\|}$and the perpendicular interface by $d s_{\perp}$. The dimensions of the unit cell are represented by $d_{\|}$and $d_{\perp}$ in the streamwise and the transverse directions respectively, as are shown in Figure 1. The width of the channel wherein the flow is in the streamwise direction is represented by $d c_{\perp}$ and the width of the channel occupied by transversely flowing fluid is represented by $d c_{\|}$. Therefore $d c_{\perp}=d_{\perp}-d s_{\perp}$ and $d c_{\|}=d_{\|}-d s_{\|}$.
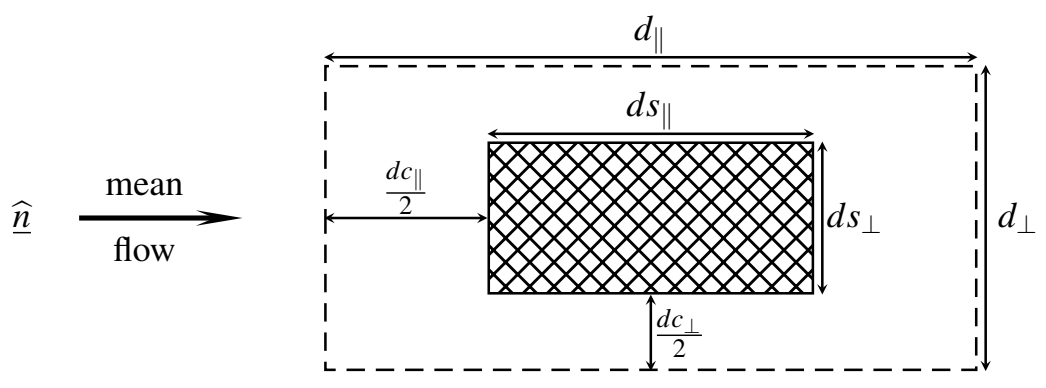

Figure 1: Notation for the unit cell with respect to the streamwise (or mean flow) direction.

The aspect ratio is defined as follows:

$$
\alpha \equiv \frac{d_{\perp}}{d_{\|}}=\frac{d s_{\perp}}{d s_{\|}}=\frac{d c_{\perp}}{d c_{\|}} .
$$

The porosity of this porous structure is then given by

$$
\varepsilon=\frac{d_{\|} d_{\perp}-d s_{\|} d s_{\perp}}{d_{\|} d_{\perp}}=1-\left(\frac{d s_{\perp}}{d_{\perp}}\right)^{2},
$$

yielding the following useful relation for the particular geometry:

$$
\frac{d s_{\perp}}{d_{\perp}}=\sqrt{1-\varepsilon} .
$$

Two different levels of staggering of the solid phase in the streamwise direction will be studied, namely a regular array and a fully staggered array as shown in Figure 2. We define as follows a parameter $\xi$ which relates to the cross-stream staggeredness of the solid material:

$$
\xi \equiv \begin{cases}0 & \text { Regular array } \\
\frac{1}{2} & \text { Fully staggered array }\end{cases}
$$




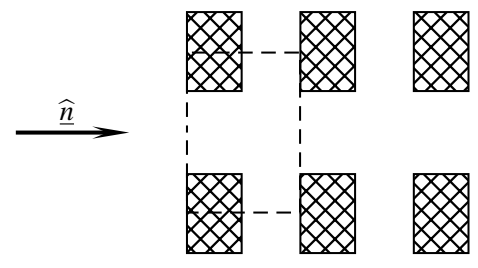

(a)

Regular array with $\varepsilon=0.75$.

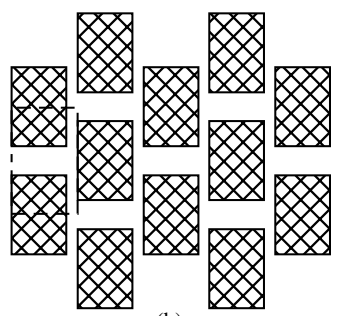

(b)

Fully staggered array with $\varepsilon \approx 0.27$.

Figure 2: An illustration of the different arrays studied, as well as typical unit cells chosen for the different cases and the streamwise direction $\underline{\hat{n}}$.

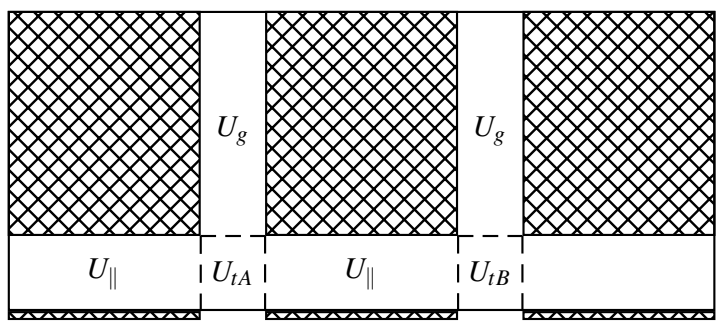

Figure 3: The model considered for a regular configuration where: $p_{U_{t A}}=p+\delta p_{\|}$ and $p_{U_{t B}}=p$.

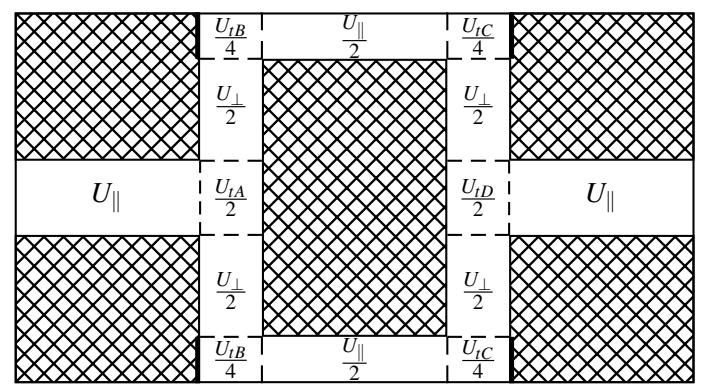

Figure 4: The model considered for a fully staggered configuration where: $p_{U_{t A}}=$ $p+\frac{1}{2} \delta p_{\perp}, p_{U_{t B}}=p, p_{U_{t C}}=p-\delta p_{\|}$and $p_{U_{t D}}=p-\delta p_{\|}-\frac{1}{2} \delta p_{\perp}$.

For the derivations of the permeability, the simplistic models shown in Figures 3 and 4 are considered. That sub-volume of a unit cell occupied by fluid flowing parallel to the net streamwise direction is given by $U_{\|}$and $U_{\perp}$ represents the volume of the transverse channel. In the transfer volume $U_{t}$, the wall shear stresses 
acting on the fluid-solid interfaces are neglected, thus the pressure drop is assumed zero. It is evident from numerous numerical calculations that the stagnation point with its large pressure causes less shear stress along those parts of $S_{o f}$ lying within the transfer volumes $U_{t}$.

An analytical model for the determination of the hydrodynamic permeability in the Darcy regime in terms of porosity was developed by Firdaouss and Du Plessis [1]. Unit cells representing different levels of streamwise staggering and different dimensions were examined and a general expression for the dimensionless permeability for these cells was obtained. This analytical model was based on a piece-wise plane Poiseuille flow approximation for interstitial flow between neighbouring particles. In their analytical model, however, the pressure gradient in the transverse channel was taken over the entire $d s_{\perp}$. In this section an identical analytical method of Firdaouss and Du Plessis [1], is used to obtain an expression for the permeability of the model presented in Figures 3 and 4.

The interstitial flow is assumed to be time independent, incompressible, Newtonian, free of body forces and, since we are interested in hydrodynamic permeability only, creep flow is assumed. The flow is thus governed by the interstitial continuity equation

$$
\nabla \cdot \underline{v}=0
$$

and the interstitial equation for creep flow

$$
\nabla p=\nabla \cdot \underline{\underline{\tau}}=\mu \nabla^{2} \underline{\underline{v}}
$$

where $\underline{v}$ is the interstitial velocity defined at each point. In a plane Poiseuille flow approximation, the wall shear stress and corresponding channel-wise pressure gradient are respectively given by

$$
\tau_{w}=\frac{6 \mu w}{d c} \quad \text { and } \quad\|-\nabla p\|=\frac{12 \mu w}{d c^{2}} .
$$

Here $w$ is the average channel velocity and $d c$ the normal distance between the facing surfaces.

The dimensionless Darcy permeability for the streamwise direction is defined as

$$
K \equiv \frac{k}{d_{\|} d_{\perp}}=\frac{\mu q}{d_{\|} d_{\perp}\|\nabla p\|}=\frac{\mu q}{d_{\perp} \delta p},
$$

where $d_{\|} d_{\perp}$ is the 'volume' of the two dimensional unit cell.

For a streamwise regular array, it follows straightforwardly that the pressure gradient, the total pressure drop and the dimensionless permeability are given by

$$
\begin{gathered}
-\nabla_{\|} p=\frac{12 \mu w_{\|}}{d c_{\perp}^{2}}=\frac{12 \mu q}{d_{\perp}^{2}(1-\sqrt{1-\varepsilon})^{3}}, \\
\delta p=\delta p_{\|}=-\nabla_{\|} p d s_{\|}
\end{gathered}
$$


and

$$
K=\frac{\alpha(1-\sqrt{1-\varepsilon})^{3}}{12 \sqrt{1-\varepsilon}}
$$

respectively, as was also obtained by Firdaouss and Du Plessis [1]. Here $\nabla_{\|}$is defined as a scalar operator in the streamwise direction.

\subsection{Streamwise staggered array}

From flux-conservation it follows for a streamwisely staggered array that

$$
w_{\perp}=\xi w_{\|} \frac{d c_{\perp}}{d c_{\|}}=\xi \alpha w_{\|} .
$$

The total pressure drop over the unit cell consists of the pressure drop in the parallel as well as the transverse channels.

$$
\delta p=\delta p_{\|}+\xi \delta p_{\perp}
$$

The pressure drop in the parallel channel is given by eqn.(10) whereas the pressure drop in the transverse channel is given by

$$
\delta p_{\perp}=-\nabla_{\perp} p\left(2 d s_{\perp}-d_{\perp}\right)
$$

where $\nabla_{\perp}$ is a scalar operator. Whence, from eqns. (1), (9) and (12), eqn. (14) reduces to

$$
\delta p_{\perp}=-\xi \alpha^{4} \nabla_{\|} p d s_{\|}\left(2-\frac{1}{\sqrt{1-\varepsilon}}\right) .
$$

From eqns. (10), (13) and (15) it there-upon follows that

$$
\delta p=-\nabla_{\|} p d s_{\|}+\xi \delta p_{\perp}=-\nabla_{\|} p d s_{\|}\left[1+\xi^{2} \alpha^{4}\left(2-\frac{1}{\sqrt{1-\varepsilon}}\right)\right] .
$$

Substituting eqns. (16) and (9) into eqn. (8) yields the following dimensionless permeability:

$$
K=\frac{\mu q}{d_{\perp} \delta p}=\frac{\alpha(1-\sqrt{1-\varepsilon})^{3}}{12\left[\sqrt{1-\varepsilon}+\xi^{2} \alpha^{4}(2 \sqrt{1-\varepsilon}-1)\right]} .
$$

This result differs from that of Firdaouss and Du Plessis [1] due to the exclusion of the part of $S_{f s \perp}$ near the stagnation points from the transfer volume $U_{t}$, as indicated on Figure 4. In this region there exists a wall shear stress as well as a negative pressure gradient opposite to the interstitial flow direction. There is thus a net force against the flow direction consisting of the positive pressure gradient and the wall shear stress. Firdaouss and Du Plessis [1] neglected the change of the direction of the pressure gradient in their direct model, and thus a much smaller net force against the interstitial flow direction (namely zero) was considered. In the derivation of eqn. (17) the wall shear stress as well as the pressure gradient on that part of $S_{f s \perp}$ was taken as zero. 


\section{Volume averaging and closure of momentum equations}

The results obtained in eqn. (17) may also be obtained more indirectly from the volume averaged transport equation, a method which is also applicable in more general cases of flow in porous media. The actual interstitial flow velocity field $\underline{v}$ can be averaged volumetrically over a Representative Elementary Volume (REV), $U_{o}$, yielding the following phase average velocity $q$, also known as the superficial velocity and of which the direction is the mean flow (or streamwise) direction used in the previous section,

$$
\underline{q} \equiv \frac{1}{U_{o}} \iiint_{U_{f}} \underline{v} d U .
$$

Volume averaging of eqns. (5) and (6) over any stationary porous structure, which has a spatially uniform porosity and an average flow which is time independent, yields respectively

$$
\nabla \cdot q=0
$$

and, if $\iint \underline{n}\langle p\rangle_{f} d S$ is assumed to be zero,

$$
S_{f s}
$$

$$
-\nabla\langle p\rangle_{f}=\frac{1}{U_{f}} \iint_{S_{f s}} \underline{n} p d S-\frac{1}{U_{f}} \iint_{S_{f s}} \mu \underline{n} \cdot \nabla_{\underline{v}} d S .
$$

Eqn. (20) may now be 'closed' for a particular porous medium by the introduction of a Rectangular Representative Unit Cell (RRUC) within which the surface integral is evaluated. The notation already established for the unit cell shown in Figure 1 will also be applicable to the RRUC. From eqn. (20) now follows that

$$
\begin{aligned}
& -\nabla\langle p\rangle_{f}=\frac{1}{U_{f}} \iint \underline{n} \overline{p_{w}} d S+\frac{d s_{\|}}{2 d_{\|} U_{f}} \iint \underline{n} \overline{p_{w}} d S+\frac{d c_{\|}}{d_{\|} U_{f}} \iint \underline{n} \overline{p_{w}} d S \\
& S_{f s \|} \quad S_{f s \perp A A} \quad S_{f s \perp B B} \\
& +\frac{d s_{\|}}{2 d_{\|} U_{f}} \iint \underline{n} \overline{p_{w}} d S+\frac{1}{U_{f}} \iint \underline{n} \widetilde{p_{w}} d S \\
& S_{f s \perp C C} \\
& -\frac{1}{U_{f}} \iint \mu \underline{n} \cdot \nabla \underline{v} d S-\frac{d c_{\|}}{d_{\|} U_{f}} \iint \mu \underline{n} \cdot \nabla \underline{v} d S \\
& S_{f s \|} \\
& S_{f s \perp B B}
\end{aligned}
$$




$$
\frac{-\frac{d s_{\|}}{2 d_{\|} U_{f}} \iint \mu \underline{n} \cdot \nabla_{S_{f s \perp A A}} \underline{\underline{v}} d S-\frac{d s_{\|}}{2 d_{\|} U_{f}} \iint \mu \underline{\underline{n}} \cdot \nabla_{S_{f s \perp C C}} \underline{\underline{v}} d S .}{}
$$

The pressure term was split into the average wall channel pressure and a wall pressure deviation. The latter term is zero, because, according to the definition of deviation, the positive and the negative parts of the deviation will cancel out on each fluid-solid interface. The integral of the average wall channel pressure is then split into an integral over the fluid-solid interface in the fluid channels parallel to the streamwise direction and one over the fluid-solid interface in the transverse fluid channels. The integral over the parallel channel will be zero, since the average wall channel pressures on the upper and the lower surfaces will be equal and thus cancel vectorially. The integral over the transverse channels is then split into three integrals which are weighed according to their relative frequency of occurrence if the RRUC is shifted in the streamwise direction. The $S_{f s \perp B B}$ term corresponds to the instances when the boundaries of the RRUC are situated in the transverse fluid channels. The $S_{f s \perp A A}$ term and the $S_{f s \perp C C}$ term correspond to the instances when the transverse boundaries of the RRUC intercept the second half of the solid phase and the first half of the solid phase, respectively. These different RRUC orientations are shown in Figure 5.
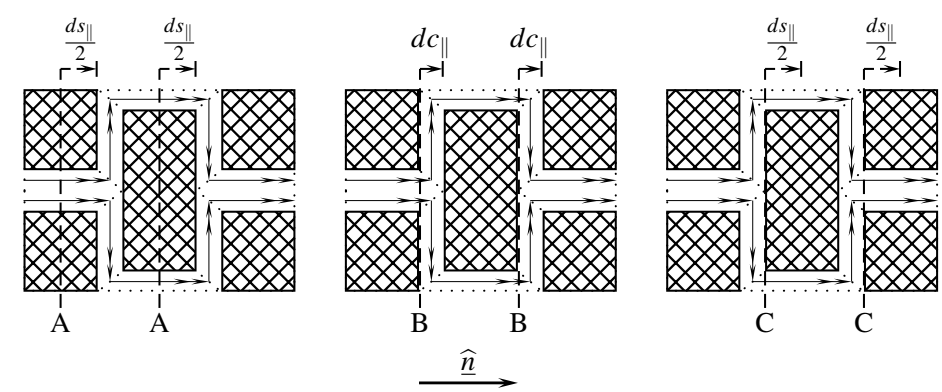

Figure 5: The shifting method of the RRUC for a fully-staggered configuration.

The shear stress integral is split in a similar manner as the pressure term. The magnitude of the wall shear stress on each fluid-solid interface in the transverse channels is equal. The $S_{f s \perp A A}, S_{f s \perp B B}$ and $S_{f s \perp C C}$ terms should thus be zero since the wall shear stresses on their two surfaces will cancel vectorially. If the solid phase was not fully staggered in the streamwise direction and the length of the transverse channel, where the interstitial flow direction is $\underline{\underline{n}}$, differs from the length of the transverse channel wherein the flow is in the $-\underline{\breve{n}}$ direction, (with $\underline{\breve{n}} \times \underline{\widehat{n}}=\underline{0}$ ) the integrals over $S_{f s \perp A A}$ and $S_{f s \perp C C}$ will not be zero respectively. If an REV is considered, there should only exist a pressure drop in the mean streamwise direction $\underline{\hat{n}}$, and the net pressure drop in the direction perpendicular to $\underline{\widehat{n}}$ should 
be zero. The net force acting on the fluid in a particular channel is zero if piecewise straight streamlines (as illustrated in Figure 5) are assumed. The magnitude of the wall shear stress times the surface area it is acting on should be equal to the magnitude of the pressure drop in that channel times its cross-sectional area. Since the net pressure drop in the transverse direction is zero, the net wall shear stress on the surfaces of those channels should also be zero. The RRUC should be a representative unit cell and therefore it is expected that, in eqn. (20), the part of the integral of the shear stress term which is taken over $S_{f s \perp}$ should be zero. Since $S_{f S \perp B B}$ is zero for all levels of streamwise staggering, the net effect of the integrals taken over $S_{f s \perp A A}$ and $S_{f s \perp C C}$ should also be zero. The wall shear stresses on the surfaces of $S_{f s \perp A A}$ will cancel vectorially with the wall shear stresses on the surfaces of $S_{f s \perp C C}$, if the two terms are weighed equally.

The underlined terms in eqn. (21) is zero. This equation thus reduces to

$$
\begin{aligned}
-\nabla\langle p\rangle_{f}= & \frac{d s_{\|}}{2 d_{\|} U_{f}} \iint \underline{n} \overline{p_{w}} d S+\frac{d c_{\|}}{d_{\|} U_{f}} \iint \underline{n} \overline{p_{w}} d S \\
& +\frac{d s_{S_{f} \perp A A}}{2 d_{\|} U_{f}} \iint \underline{n} \underline{\underline{p_{w}}} d S-\frac{1}{U_{f}} \iint \mu \underline{n} \cdot \nabla \underline{\widetilde{v}} d S \\
S_{f_{s} \perp C C} &
\end{aligned}
$$

and substitutions according to the geometric assumptions yield:

$$
\begin{aligned}
-\nabla\langle p\rangle_{f} & =\xi \delta p_{\perp}\left[\frac{d c_{\|} d_{\perp}+d s_{\|} d c_{\perp}}{d_{\|} U_{f}}\right]+\delta p_{\|}\left[\frac{d s_{\perp} d c_{\|}}{d_{\|} U_{f}}\right]+\frac{\tau_{\|} S_{\|}}{U_{f}} \\
& =\left[\frac{\xi \delta p_{\perp}+\delta p_{\|}}{d_{\|}}\right],
\end{aligned}
$$

which exactly corresponds with eqn. (13), used in the derivation of the dimensionless permeability with the direct method. The gradient of the intrinsic phase average of the pressure can also be written in terms of the shear stresses in the parallel and the transverse channels. It then follows from eqn. (22) that

$$
-\nabla\langle p\rangle_{f}=\frac{1}{d_{\|} d c_{\perp}}\left[\tau_{\|} S_{\|}+\alpha \xi \tau_{\perp} S_{\perp}\right] \underline{\underline{n}}
$$

where $\tau_{\perp} S_{\perp}=\delta p_{\perp} d c_{\|}$and $S_{\perp}=2\left(d s_{\perp}-d c_{\perp}\right)$.

It is assumed that plane Poiseuille flow is a good approximation for the interstitial flow in the channels between the solid particles for this two dimensional case study. We thus have the following expressions for the shear forces in the transverse and the streamwise channels respectively:

$$
\tau_{\perp} S_{\perp}=\frac{6 \mu w_{\perp}}{d c_{\|}}\left(4 d s_{\perp}-2 d_{\perp}\right) \quad \text { and } \quad \tau_{\|} S_{\|}=\frac{6 \mu w_{\|}}{d c_{\perp}}\left(2 d s_{\|}\right) .
$$


The interstitial velocity relation is given by

$$
\frac{w_{\perp}}{w_{\|}}=\xi \alpha .
$$

From eqn. (24) it then follows that

$$
-\nabla\langle p\rangle_{f}=\frac{12 \mu q d_{\perp}}{d c_{\perp}^{3} d_{\|}}\left[d s_{\|}+\alpha^{4} \xi^{2}\left(2 d s_{\|}-d_{\|}\right)\right] \underline{\underline{n}} .
$$

From the definition of the dimensionless Darcy permeability for the streamwise direction it there-upon follows that

$$
K \equiv \frac{\mu q}{U_{o}\left\|\nabla\langle p\rangle_{f}\right\| \overline{\overline{12} d_{\perp}^{2} d_{\|}\left[d s_{\|}+\alpha^{4} \xi^{2}\left(2 d s_{\|}-d_{\|}\right)\right]}} .
$$

After substitutions, this expression, obtained by means of volume averaging, is identical to expression (17) where the permeability was obtained directly without involving volume averaging.

\section{Discussion}

Eqn. (24) corresponds to the following equation obtained by Lloyd et al. [3] (their equation (15)),

$$
-\nabla\langle p\rangle_{o}=\frac{U_{f}}{U_{\|}+U_{t}} \cdot \frac{\tau_{\|} S_{\|}+\xi \tau_{\perp} S_{\perp}}{U_{o}},
$$

where squares rather than rectangles were considered and the aspect ratio, $\alpha$, was thus set equal to 1 . Note that $S_{\perp}$ in eqn. (29) is $2 d c_{\perp}$ larger than $S_{\perp}$ in eqn. (24) due to the assumption of the present model.

In a staggered configuration the dimensionless permeability obtained is defined only for porosities up to the point $d s_{\perp}=d c_{\perp}$ where subsequent rectangles cease to overlap. Note that the denominator of eqn. (17) is zero or negative if

$$
\varepsilon \geq \frac{\left(3 \alpha^{4} \xi^{2}+1\right)\left(\alpha^{4} \xi^{2}+1\right)}{\left(2 \alpha^{4} \xi^{2}+1\right)^{2}},
$$

where the lower bound of the RHS of eqn. (30) is 0.75 when $\alpha$ tends to infinity.

In the following example expression (17) is compared with the numerical results obtained by Firdaouss and Du Plessis [1] as well as their analytical results (which is identical to the analytical results obtained by Lloyd et al. [3] if $\alpha=1$ ). In this example, the aspect ratio is 4 and thus the tortuosity in the streamwise direction is

$$
\chi=\frac{L_{e}}{L}=1+\xi \alpha \sqrt{1-\varepsilon}=1+2 \sqrt{1-\varepsilon} .
$$




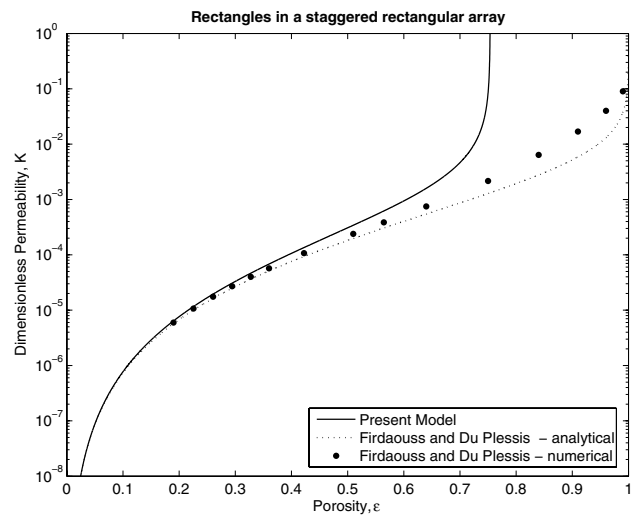

Figure 6: Streamwise staggered array with $\alpha=4$.

\section{Conclusions}

A new pore-scale model was introduced to predict the hydrodynamic permeability of low porosity configurations of rectangles. The results closely correlate with numerical computations reported in literature. Important outcomes of this study are the identical results obtained by the direct approach and by the volumetric averaging coupled with closure by a pore-scale model, even in the case of high aspect ratio solid parts and high tortuosities.

\section{References}

[1] Firdaouss, M. \& Du Plessis, J.P., On the prediction of Darcy permeability in non-isotropic periodic two-dimensional porous media, Journal of Porous Media, 7(2), pp. 119-131, 2004.

[2] Lloyd, C.A., Hydrodynamic permeability of staggered and non-staggered regular arrays of squares, MSc Thesis, University of Stellenbosch, South Africa, 2003.

[3] Lloyd, C.A., Du Plessis, J.P. \& Halvorsen, B.M., On closure modelling of volume averaged equations for flow through two-dimensional arrays of squares, Advances in Fluid Mechanics, vol. V, A. Mendes, M. Rahman \& C.A. Brebbia (Eds), Proc. of the International Conference on Advances in Fluid Mechanics AFM2004, Lisbon, Portugal, May 2004, pp. 85-93.

[4] Whitaker, S., The Method of Volume Averaging, Theory and Applications of Transport in Porous Media, 13, Kluwer Academic Publishers, 1999. 